\title{
Un régulateur de la croissance et de la taille cellulaire chez la drosophile
}

Pour de nombreuses espèces animales, le programme de croissance mis en jeu lors du développement s'arrête à l'âge adulte. La taille définitive des organes doit donc être soumise à un mécanisme de contrôle. $\mathrm{Au}$ cours du développement, l'augmentation de la masse tissulaire est la résultante de deux mécanismes étroitement intriqués, la croissance cellulaire (augmentation de la taille des cellules) et la prolifération cellulaire (augmentation du nombre de cellules). Ces deux processus peuvent être dissociés chez la levure, comme le montre l'identification de deux classes distinctes de mutants: les uns bloquent la croissance cellulaire, provoquant secondairement un arrêt de leur prolifération (voir ci-dessus), alors que les autres bloquent la prolifération des cellules sans arrêter leur croissance [1]. De même, chez la drosophile (mouche du vinaigre), des travaux récents indiquent que la croissance des cellules est réglée indépendamment de leur prolifération $[2,3]$. En effet, si la division cellulaire est bloquée, la taille des tissus est la même, mais les cellules, moins nombreuses, sont plus grosses. La croissance des cellules est un élément déterminant de leur prolifération puisqu'elles doivent augmenter en taille avant de se diviser: ainsi, un cycle classique de division cellulaire se déroule selon la séquence suivante : une étape postmitotique (G1), la synthèse d'ADN (S), une étape prémitotique (G2), et la mitose (M). L'augmentation de la taille des cellules a lieu durant toutes les phases intermitotiques (G1, S et G2), mais il est crucial que, avant d'amorcer la synthèse d'ADN, une cellule ait atteint une taille critique [3]. L'augmentation du nombre de ribosomes constitue un des événements-clés lors de la croissance des cellules [4]. Comment la fixation d'un facteur de croissance extracellulaire sur son récepteur membranaire peut-elle induire une augmentation de la biosynthèse des ribosomes dans le cytoplasme? Dans le cas d'une stimulation par l'insuline [4], la fixation de l'hormone sur son récepteur déclenche une cascade de réactions intracellulaires (figure 1) dont une conséquence est l'activation de la S6kinase, ainsi nommée parce qu'elle phosphoryle la protéine ribosomique $\mathrm{S} 6$. On observe alors une augmentation de la traduction d'une sous-famille d'ARN messagers codant pour les protéines ribosomiques et les facteurs d'amorçage de la traduction. Ainsi, l'activation de cellules par des facteurs de croissance provoque, par l'intermédiaire de la S6kinase, une augmentation de la traduction des protéines nécessaires à la synthèse protéique.

La drosophile constitue un outil génétique puissant pour analyser le rôle physiologique d'une protéine, en particulier pour l'étude des relations entre croissance et prolifération cellulaire [5]. Pour cette raison, nous avons identifié, chez la drosophile, l'homologue fonctionnel de la S6kinase (DS6K) et engendré des drosophiles mutantes chez lesquelles DS6K est inactivée [6]. La plupart de ces mutants meurent au cours du développement, et les survivants mettent 14 jours pour atteindre l'âge adulte, alors que les mouches sauvages se développent en neuf jours. La taille des mouches adultes mutantes est considérablement réduite (de $40 \%$ à $50 \%$ ) du fait d'une diminution de la taille des cellules, mais sans que leur nombre ne soit significativement affecté. Ces observations ayant été faites sur les tissus externes de la mouche adulte dont la croissance est terminée, il était important d'étudier également les tissus en cours de prolifération. Chez la drosophile, les tissus adultes dérivent des disques imaginaux qui sont des amas de cellules individualisés dès le stade embryonnaire. Les disques imaginaux prolifèrent et leur taille augmente pendant la vie larvaire et le début de la métamorphose, puis ils se différencient. L'analyse des disques imaginaux de larves mutantes pour DS6K a montré, d'une part, un ralentissement de toutes les étapes du cycle cellulaire (expliquant le retard de développement) et, d'autre part, une diminution de la taille des cellules qui prolifèrent (expliquant la diminution de la taille des mouches adultes). Ces résultats montrent que DS6K est un régulateur de la croissance cellulaire et de la taille des cellules [6].

A ce jour, la mutation DS6K constitue, chez la drosophile, le seul exemple de mutant pour lequel la réduction de la taille des cellules ne s'accompagne pas d'une diminution de leur nombre. En effet, pour obtenir des mouches de petite taille, il suffit de restreindre la nourriture des larves, ce qui entraîne principalement une diminution du nombre de cellules [7]. De même, les drosophiles mutantes soit pour le substrat du récepteur de l'insuline (IRS), soit pour la PI3K (phospho-inositoside 3 kinase), tous deux situés en amont de DS6K dans la voie de signalisation du récepteur de l'insuline (figure 1), ont une taille réduite, conséquence d'un effet à la fois sur la taille et le nombre des cellules $[8,9]$. Puisque l'activation de DSK6 augmente la synthèse des ribosomes, on pouvait sup- 


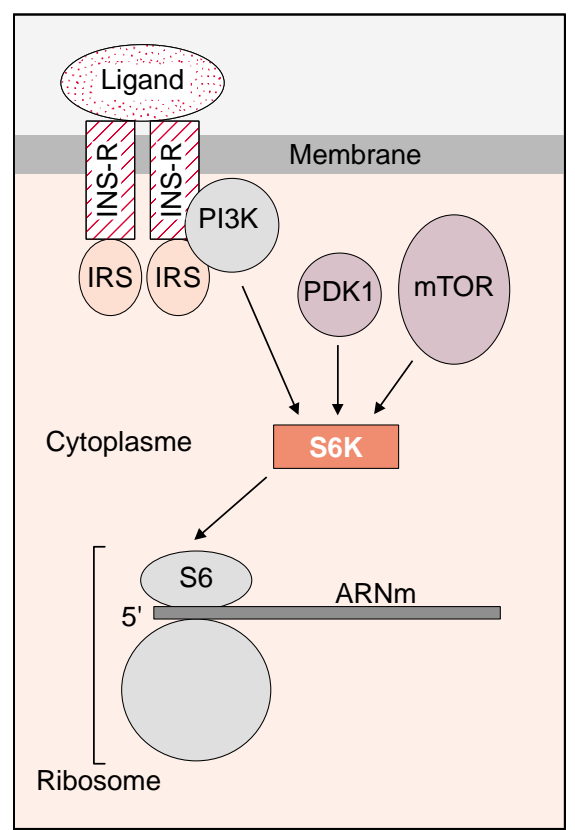

Figure 1. Localisation de la S6kinase sur la voie de signalisation de l'insuline. La fixation de l'insuline ou d'une molécule apparentée, sur son récepteur (INS-R) entraîne la phosphorylation d'un substrat appelé IRS (insulin receptor substrate) et le recrutement à la membrane de la PI3K (phosphoinositoside 3 kinase). Une des conséquences de l'activation de la PI3K est la phosphorylation de la S6kinase (S6K) qui provoque une augmentation de la traduction des ARN messagers (ARNm) codant pour les protéines ribosomiques. Les protéines $P D K 1$ (protein dependent kinase 1) et mTOR (mammalian target of rapamycin) participent également à l'activation de la S6kinase. La succession des intermédiaires de cette voie de signalisation, en aval de la PI3K, n'est pas encore bien définie. poser que le nombre de ribosomes soit directement impliqué dans la régulation de la croissance et de la taille cellulaire. Chez la drosophile, les mutations entraînant une diminition de la production de ribosomes induisent effectivement un retard de développement, du fait d'un ralentissement de la croissance des cellules et du cycle cellulaire [10]. Cependant, ni la taille de ces drosophiles mutantes adultes, ni celle de leurs cellules ne sont affectées [6]. Ces résultats indiquent que le déficit en ribosomes pourrait être impliqué dans le retard de développement observé chez les drosophiles déficientes en DS6K, mais qu'il n'est pas, en revanche, directement impliqué dans la régulation de la taille des cellules. Il reste donc à identifier le mécanisme par lequel DS6K règle la taille des cellules.

En conclusion, il apparaît que les mécanismes de contrôle de la taille et de la croissance cellulaire chez la drosophile utilisent des régulateurs communs tels que DS6K [6] ou IRS [8], et agissent secondairement sur la prolifération. Ces molécules sont des intermédiaires de la voie de signalisation du récepteur de l'insuline qui est conservée entre les mammifères et la drosophile. Ainsi, chez la souris, il existe deux gènes codant pour deux S6kinases très similaires [11]. L'inactivation de l'une d'elles provoque un retard de croissance [11], mais augmente l'expression de l'autre. Par conséquent, seule l'inactivation des deux kinases devrait permettre de savoir si la taille des cellules de mammifères est également contrôlée par la S6kinase. Les développements futurs de ces travaux chez les mammi- fères, mais aussi chez la drosophile, devraient aider à comprendre les anomalies de régulation de la croissance et de la prolifération cellulaire, observées dans les processus de cancérisation.

1. Johnston GC, Pringle JR, Hartwell LH. Coordination of growth with cell division in the yeast $S a c$ charomyces cerevisiae. Exp Cell Res 1977; 105: 79-98.

2. Conlon I, Raff M. Size control in animal development. Cell 1999; 96: 235-44.

3. Neufeld TP, de la Cruz AF, Johnston LA, Edgar BA. Coordination of growth and cell division in the Drosophila wing. Cell 1998; 93: 1183-93.

4. Thomas G, Hall MN. TOR signalling and control of cell growth. Curr Opin Cell Biol 1997; 9 : 782-7.

5. Perrin L, Arquier N, Sémériva M. Les relations entre croissance et prolifération reconsidérées chez la drosophile. Med Sci 1999; 15: 1105-17. 6. Montagne J, Stewart MJ, Stocker H, Hafen E, Kozma SC, Thomas G. Drosophila S6 Kinase: a regulator of cell size. Science 1999; 285: 2126-9.

7. Simpson P. Parameters of cell competition in the compartments of the wing disc of Drosophila. Dev Biol 1979; 69: 182-93.

8. Bohni R, Riesgo-Escovar J, Oldham S, et al. Autonomous control of cell and organ size by CHICO, a Drosophila homolog of vertebrate IRS1-4. Cell 1999; 97: 865-75

9. Weinkove D, Neufeld TP, Twardzik T, Waterfield MD, Leevers SJ. Regulation of imaginal disc cell size, cell number and organ size by Drosophila class I(A) phosphoinositide 3-kinase and its adaptor. Curr Biol 1999; 23: 1019-29.

10. Lambertsson A. The minute genes in Drosophila and their molecular functions. Adv Genet 1998; 38: 69-134.

11. Shima H, Pende M, Chen Y, Fumagalli S, Thomas G, Kozma SC. Disruption of the $\mathrm{p} 70(\mathrm{~s} 6 \mathrm{k}) / \mathrm{p} 85(\mathrm{~s} 6 \mathrm{k})$ gene reveals a small mouse phenotype and a new functional $\mathrm{S} 6$ kinase. $E M B O J$ 1998; 17: 6649-59.

\section{Jacques Montagne}

Friedrich Miescher-Institut, Maulbeerstrasse 66, CH-4058 Bâle, Suisse. 